\title{
Somatosensory prior entry
}

\author{
Mark J. YATES ANd Michael E. R. Nicholls \\ University of Melbourne, Melbourne, Victoria, Australia
}

\begin{abstract}
The perceived timing of sensory events does not necessarily match the actual timing. In the present study, we investigated the effect of location of attention on the perceived timing of somatosensory stimuli. Participants judged the temporal order of two taps, delivered one to each hand, with taps presented at different elevations (upper and lower) on the opposing hands. The task was to discriminate the elevation of the tap presented first (or second). This vertical discrimination task was orthogonal to the horizontal attentional cuing manipulation, removing the response bias confound that has undermined earlier studies investigating the impact of attention on the perceived timing of sensory stimuli. We manipulated spatial attention either (1) exogenously (Experiment 1) in 13 participants, using brief taps to either the left or right hand, or (2) endogenously (Experiment 2) in 22 participants, using centrally presented symbolic cues. The results supported the hypothesis that attended stimuli are perceived more rapidly than unattended stimuli. This effect was larger when attention was exogenously manipulated. Previous research has demonstrated a similar effect for visual stimuli. The present study, which extends this result to somatosensory perception, indicates that the phenomenon may represent a more global feature of the perceptual system, which is possibly mediated by a common modality-independent mechanism.
\end{abstract}

Our senses provide many forms of information to the brain. One of the most fundamental categories of sensory data is the timing (e.g., onset, duration, order, rate) of stimuli in the environment. Our brains use this timing information to perceive the environment around us. An illustrative example is the small time difference in the arrival of soundwaves from a singular sound source to the left and right ears, which is used by the brain to locate the source of the sound (Yamada, Kaga, Uno, \& Shindo, 1996). The brain's use of temporal information extends into many other aspects of perception, from the comprehension of speech to the determination of the direction and velocity of objects in the environment, the integration of sensory information from different modalities, the location of the body in space (Ehrsson, Spence, \& Passingham, 2004), and the ability to distinguish between self-caused versus externally caused events in the environment (Cunningham, Billock, \& Tsou, 2001; Wegner \& Wheatley, 1999). Despite the ubiquity and usefulness of sensory temporal information, the way in which the brain codes this information is largely unknown.

A significant gap in our knowledge in this area is a basic account of the relationship between the actual timing of stimulus events and the perceived timing of those events, the factors that influence this relationship, and the manner (i.e., where, when, and how) in which this influence occurs. Central to such an account is the role of attention, particularly in light of the opportunity that attention presents in "connecting the mental level of description of processes used in cognitive science with the anatomical level common in neuroscience" (Posner \& Petersen,
1990, p. 25). Can paying attention to a stimulus affect the time at which that stimulus is perceived, and if so, how? One influential claim concerning the nexus between attention and perceptual latency has been that attending to a stimulus accelerates its perception: "The object of attention comes to consciousness more quickly than the objects which we are not attending to" (Titchener, 1908, p. 251). This claim is known as the doctrine of prior entry.

The question of how attention can affect the perceived timing of events is of interest both clinically and experimentally. Clinically, Rorden, Mattingley, Karnath, and Driver (1997) showed that patients with left-sided neglect - a failure to attend and orient to the left side of space - had dramatic abnormalities in the time that they perceived stimuli in the neglected hemifield, with significantly delayed perception of such stimuli as compared with the perception of stimuli presented in the normal hemifield. Within the experimental realm, the uncertain influence of attention on subjective timing obscures our understanding of the relationship between brain processes and perceptual events. This has implications for research in many areas of perception and cognition in which subjective timing measures are used (for a review, see Spence, Shore, \& Klein, 2001).

Empirical investigation of the prior entry hypothesis has yielded evidence both for (Hikosaka, Miyauchi, \& Shimojo, 1993; Rorden et al., 1997; Stelmach \& Herdman, 1991) and against (Cairney, 1975; Jaskowski, 1993; Vanderhaeghen \& Bertelson, 1974) prior entry. There has been considerable criticism of much of this work (see, e.g., Pashler, 1998; Schneider \& Bavelier, 2003), casting doubt 
on studies both in favor of, and against, the existence of prior entry. These criticisms, together with the means for addressing them, are described below.

Criticism of studies that have concluded that attention does not accelerate perception is confined to one objection: To disprove prior entry, it is necessary to show that the prior entry effect is not present under circumstances in which attention has been successfully manipulated. Some previous studies do not demonstrate the latter (e.g., the endogenous attention condition in Schneider \& Bavelier, 2003). This objection can be addressed by incorporating a simple reaction time task embedded into the experiment to provide an independent means of assessing the effectiveness of the attentional cuing (see, e.g., Vanderhaeghen $\&$ Bertelson, 1974). It should be noted, however, that although the presence of such a measure in studies of prior entry is preferable to its absence, its interpretation is not without difficulties (see Spence et al., 2001, for a discussion of the response bias confound and priming accounts of changes in simple detection latencies).

Criticism of studies supporting prior entry is more varied, but the different objections share common ground in asserting that the apparent demonstration of prior entry in these studies can be explained in full, or in part, by alternative accounts. These accounts fall into one of two categories. Either they propose that attended stimuli are indeed perceived earlier than unattended stimuli, but that this may be due to factors other than attention, or, alternatively, they propose that the purported earlier perception of attended stimuli in these studies is illusory, or at least equally attributable to nonperceptual factors.

Within the first category of alternative explanations of prior entry is the suggestion that the effect is due to peripheral within-modality sensory interactions between the cue designed to attract attention and the subsequently presented target stimulus at that location (Schneider \& Bavelier, 2003). This explanation applies only to exogenous cuing of spatial attention, which elicits automatic shifts of attention, and it can be addressed by the use of endogenous cuing, in which the shifts of attention are internally generated. The other explanation of an apparent prior entry effect within the first category is that attended stimuli are perceived earlier as a consequence of temporal attraction between the cue and target, not because they are attended (Schneider \& Bavelier, 2003); that is, the cue and target are perceived closer together in time than they actually are if they are sufficiently close together in space (Bertelson \& Aschersleben, 2003). Unlike in the previous explanation based on peripheral sensory interactions between cue and target, in this account, the shift in perceived latency is proposed to operate centrally, after sensory signals have reached the brain. It can also be addressed by the use of endogenous cuing, in which there is no cue sufficiently spatially or temporally proximate to produce the temporal attraction effect.

In alternative explanations of prior entry that fall into the second category, it is argued that the results can be explained, at least in part, by postperceptual processes - an umbrella term to incorporate response bias, response prim- ing, and shifts in decision criteria (Pashler, 1998; Shore, Spence, \& Klein, 2001; Spence et al., 2001). Stelmach and Herdman's (1991) study is illustrative. In that study, participants were presented with two dots, one dot to the left, and one dot to the right of a screen. The dots were presented separated by varying time intervals. The participants were instructed to judge which dot appeared first. Attention was directed to the left, to the right, or centrally. If attended stimuli are perceived more rapidly, then the point of subjective simultaneity (PSS) of the two dots should shift under the different spatial attention conditions. Although this is true, PSS values are not observed directly; rather, they are inferred from the point of maximal uncertainty - the point at which the proportion of left-first responses equals the proportion of right-first responses. Accordingly, shifts in the inferred PSS may not reflect genuine PSS shifts. This may occur, for example, if there is a response bias. It might be the case that directing a participant's attention to, say, the left side may bias them to select the left-first response more often while having no influence on the time at which the stimuli are perceived. This effect may be particularly pronounced when the task is difficult.

Attention-based alternative explanations of prior entry present a challenge, but improvements in experimental design can help in managing these potential confounds. An important advance has been the development of the orthogonal cuing and response dimensions methodology (see, e.g., Shore et al., 2001). Rather than observers receiving one left and one right stimulus and judging their temporal order following a left-right attentional manipulation, they are instead presented with two stimuli (as before, one to the left and one to the right), which also differ on a new dimension. In the case of the Shore et al. study, one of the stimuli was a vertical line and one a horizontal line, but there was no systematic relationship between line orientation and the side on which it appeared. The task was to judge which line orientation came first (or second). Doing this addresses the problem of response bias. With such a design, the response dimension (orientation) is orthogonal to the attentional cuing dimension (side), which minimizes or eliminates response bias, since "there is no obvious reason why 'horizontal' or 'vertical' responses should be preferentially activated by a left or right cue (whereas it is easy to see that 'left' and 'right' responses might be)" (Shore et al., 2001, p. 205).

Recent investigations into prior entry have incorporated methodological improvements such as those mentioned above and, consequently, have provided the most robust evidence to date for the existence of prior entry. In some of these more recent studies, the emphasis has been on modality-based prior entry-investigating whether focusing attention on a particular modality can accelerate the perception of stimuli in that modality (Zampini et al., 2007; Zampini, Shore, \& Spence, 2005). Methodologically rigorous investigations into spatial prior entry (accelerated perception of spatially attended stimuli) have been sparse, despite the fact that such studies provide the opportunity to understand what is arguably the more fundamental form of prior entry-prior entry operat- 
ing within a single modality. Furthermore, the studies to date have tended to focus on vision (see, e.g., McDonald, Teder-Sälejärvi, Di Russo, \& Hillyard, 2005; Shore et al., 2001). One study (Spence et al., 2001) reported a specific spatial prior entry effect for tactile and visual stimuli; however, this study was limited in a number of important ways. First, it only investigated an influence on perceived timing for endogenous shifts of attention. Second, it did not assess and control for second-order response bias (see the introduction for Experiment 1). Third, by virtue of the experimental design, it was not possible to determine whether the effect was mediated by visual, tactile, or cross-modal shifts in spatial attention. Consequently, the important question of whether (spatial) prior entry is a phenomenon that is confined to vision, or instead reflects a more general feature of the brain - independent of the sensory modality of the stimuli-is one that has not been adequately addressed by the literature to date.

The present study will explore the scope of the prior entry effect by investigating whether prior entry is present within touch, using an experimental design adapted from Shore et al. (2001). Touch is of particular interest as a modality, since on the one hand, there are grounds to anticipate that the effect of attention on perceptual latency in touch may be different from that in the other senses. Somatosensory signals can reach the cortex with particularly short latencies (Miura, Sonoo, \& Shimizu, 2003), which may affect whether prior entry occurs. On the other hand, there is now a well-established understanding of attention as an integrated multimodal network with substantial supramodal operation that involves shared cortical and subcortical neural circuitry across the different modalities (Downar, Crawley, Mikulis, \& Davis, 2000; Spence \& Driver, 2004), with the implication that many attention-related phenomena observed within one modality may extend to the other modalities.

\section{EXPERIMENT 1 Exogenous Cuing}

The location of spatial attention can be manipulated using exogenous or endogenous cues. There is increasing evidence that these two forms of attentional orienting operate differently, with different neural substrates and divergent perceptual and behavioral effects at attended locations (Friedrich, Egly, Rafal, \& Beck, 1998; Funes, Lupiáñez, \& Milliken, 2007; Hein, Rolke, \& Ulrich, 2006; Kincade, Abrams, Astafiev, Shulman, \& Corbetta, 2005). Consequently, the effect of these two forms of cuing on perceived timing may also differ, and they must be considered separately in order to isolate their individual contributions. Exogenous cuing of attention has been shown in previous studies in other modalities (see, e.g., Shore et al., 2001) to elicit larger prior entry effects, and this was examined first.

To remove the effects of response bias, we employed a design involving orthogonal cuing and response dimensions. It was still possible, however, that participants' responses might be affected by a "second-order response bias" (Shore $\&$ Spence, 2005, p. 92). In the Shore et al. (2001) study of prior entry within vision, this was the possibility that participants preferentially responded with the orientation of the line stimulus on the cued side. In the present study, it was the possibility that participants would tend to respond with the elevation of the cued tap (see the Procedure section below). This potential bias was addressed in the same manner as it was in Shore et al., by dividing participants into two groups with opposing task instructions. In this study, one group judged which tap (upper or lower) came first; the other group judged which tap came second. Any secondorder response bias should have shifted the inferred PSS in opposing directions for these two differing task conditions, canceling out any bias when the two groups were averaged, as well as providing a measure of the magnitude of the effect, which can be calculated from the differences in PSS values for the different task instructions.

To ensure that attention was manipulated effectively, a reaction time task was embedded within the temporal order judgment experiment, providing an independent behavioral measure of attentional orienting (as was described earlier).

Another methodological feature was the use of both unilateral and bilateral pairs of taps. Unilateral pairs (upper and lower taps on the same hand) cannot be used to test the prior entry hypothesis. This is because testing the hypothesis requires that there be one attended and one unattended tap, whereas unilaterally presented taps would be either both attended or both unattended. Unilateral trials were included for reasons relating to Experiment 2, in which endogenous attention was manipulated by spatially predictive cues. This required the use of unilateral trials, which were included in Experiment 1 to maintain consistency across the two experiments (see Shore et al., 2001).

\section{Method}

Participants. Thirteen healthy students took part in this experiment in return for course credit at the University of Melbourne. The complete data sets (both the reaction time and the temporal order judgment data) were excluded for 3 participants because of a failure to achieve an accuracy threshold, which was $70 \%$ (or greater) correct for the temporal order judgments. All remaining participants (3 men, 7 women) except for 1 were strongly right-handed according to the Edinburgh Inventory (Oldfield, 1971). The present study received approval from the University of Melbourne Ethics Committee, and all participants gave informed, written consent prior to participating in the study.

Apparatus and Stimuli. The experiment was conducted in a darkened, sound-attenuated room. Participants sat at a desk facing a monitor interfaced with a PC computer. Two vertical stands provided hand-grips that were $600 \mathrm{~mm}$ apart. The participants' hands were placed on ipsilateral hand grips and positioned so that the index fingers of both hands were located directly above the thumbs of the same hand. Vertical separation between the tip of the index finger and the tip of the thumb was $100 \mathrm{~mm}$. Plastic vibrotactile stimulators ("tappers," C-2 tactors, Engineering Acoustics, Inc.) were secured to the thumb, index finger, ring finger, and fifth finger of each hand, with the vibrating surfaces of the device in contact with the fingerpads/thumbpads (see Figure 1). Activation of the tappers was controlled by an interface card with a millisecond timer (Blue Chip Technology DCM-16 Digital Interface Card).

Three different classes of stimuli were administered during the experiment: cues, temporal order taps (target taps), and reaction time 


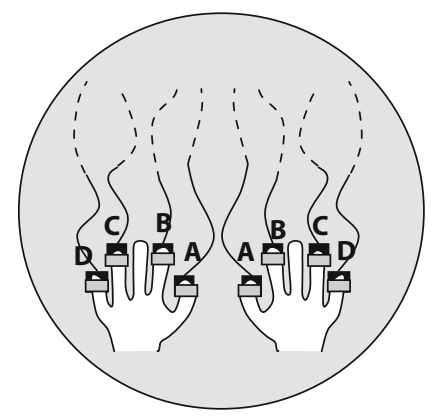

\begin{tabular}{|llr|}
\hline Digit & Tap Type & Duration \\
\hline Thumb (A) & Target tap (lower tap) & $10 \mathrm{msec}$ \\
Index finger (B) & Target tap (upper tap) & $10 \mathrm{msec}$ \\
Fourth finger (C) & Reaction time probe & $200 \mathrm{msec}$ \\
Fifth finger (D) & Exogenous cuing tap & $20 \mathrm{msec}$ \\
\hline
\end{tabular}

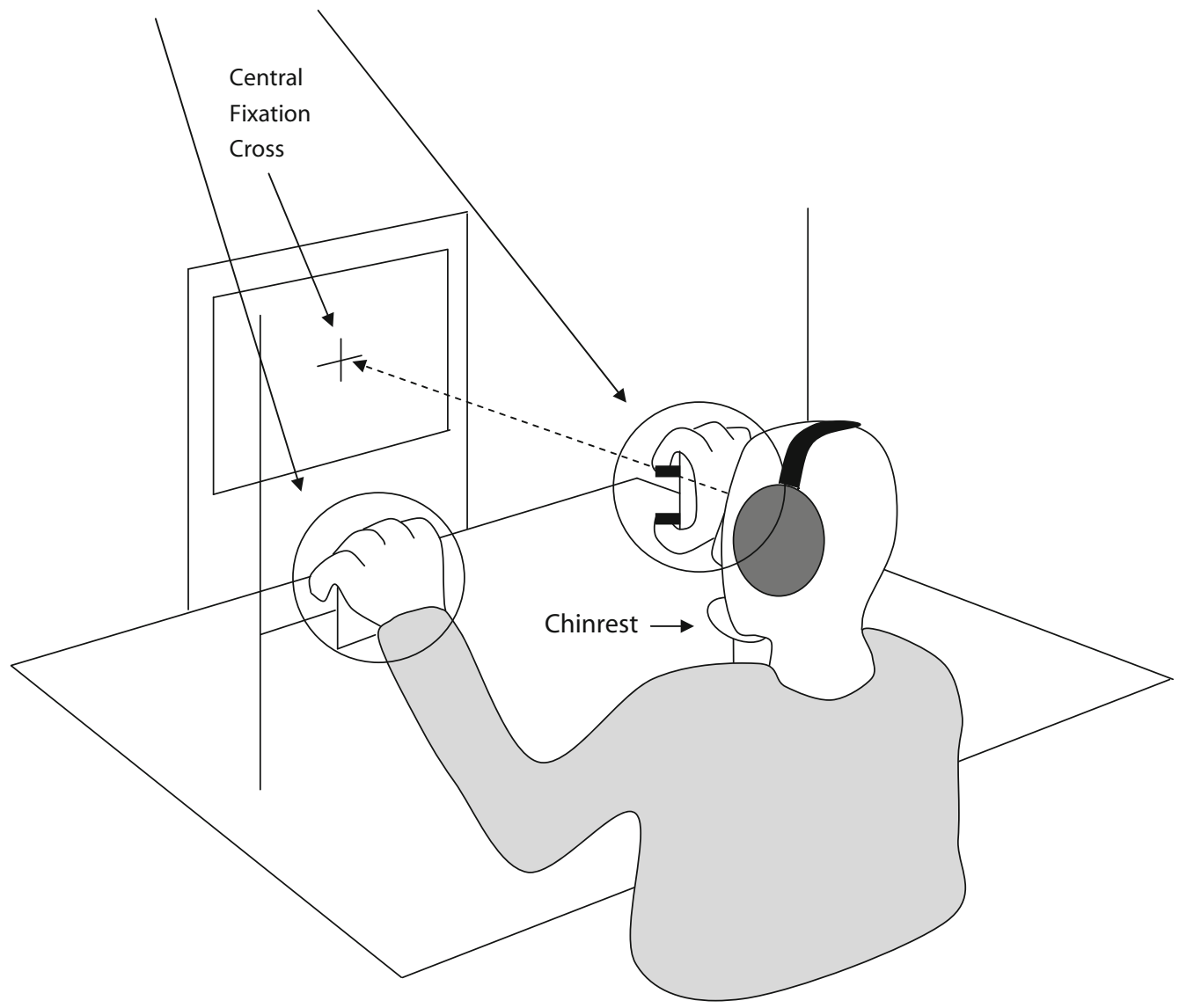

Figure 1. Schematic view of the apparatus and the participants' posture during trials. Enlarged inset shows placement of the tappers (colored black) on the fingers and thumbs of each hand, secured by fasteners (colored gray). The inset shows hands in extended posture; the actual grip posture is shown in the main diagram. Response pedals were located under the desk.

probes. Cues consisted of brief $(20 \mathrm{msec})$ bursts of vibrotactile stimulation $(250 \mathrm{~Hz})$ delivered via activation of the tappers secured to the fifth finger of the left hand, right hand, or both hands. Temporal order taps consisted of a brief $(10 \mathrm{msec})$ vibration of tappers secured to the index finger of one hand and to the thumb of the other hand. These brief bursts of vibration from the tappers resulted in a sensation similar to receiving a light tap on the stimulated finger. Reaction time probes consisted of three pulses of vibration, with each pulse lasting $40 \mathrm{msec}$ and being separated by $40 \mathrm{msec}$ (total duration of $200 \mathrm{msec}$ ) from tappers secured to the fourth finger of either hand. This was experienced by participants as a rough tactile "buzz" with discernible pulses. Throughout the experiment, white noise at $70 \mathrm{~dB}$ was presented via headphones to mask any noise made by the operation of the tappers and any extraneous noise. Additionally, the vibration of the tappers was not visually detectable. Consequently, perception of the stimuli in this experiment was attributable to detection solely through the somatosensory modality.

Procedure. Refer to Figure 2. A trial consisted of the presentation of a white central fixation cross against a black background on the computer screen, which remained present for the duration of the trial. After $300 \mathrm{msec}$, a cue to attract attention was delivered. The neutral cue condition (divided attention condition with cues on both sides) provided an appropriate baseline, which should be intermediate between the upper and lower tap cuing conditions, while holding constant the alerting effect due to cuing and the orienting of attention in the temporal dimension (Coull \& Nobre, 1998) across all three conditions. Cues were randomly presented with equal frequency and were spatially uninformative. 
Stage 1-Cuing

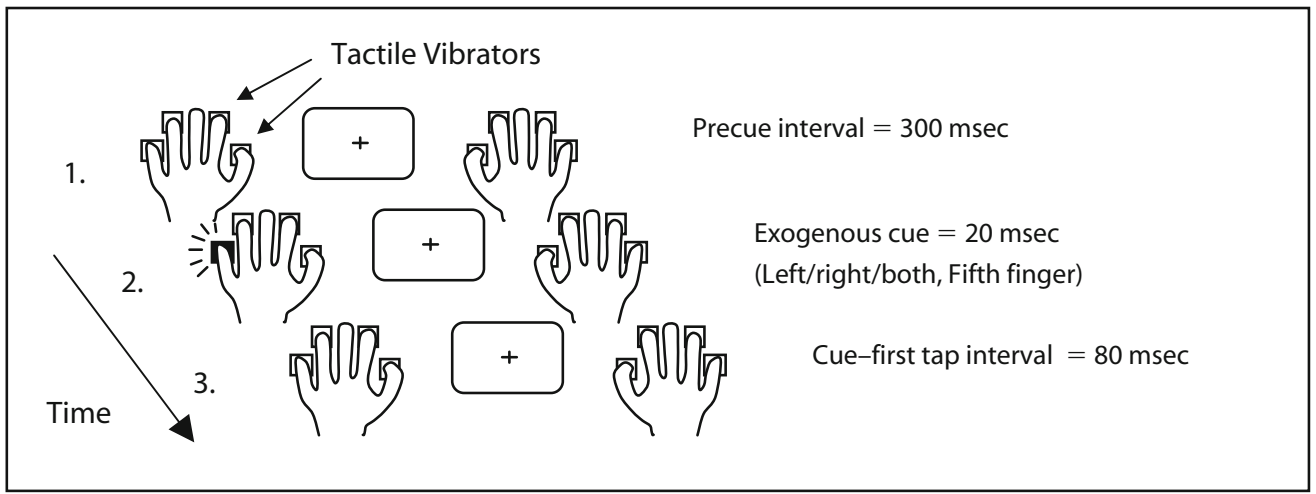

Stage 2-Experimental Stimuli
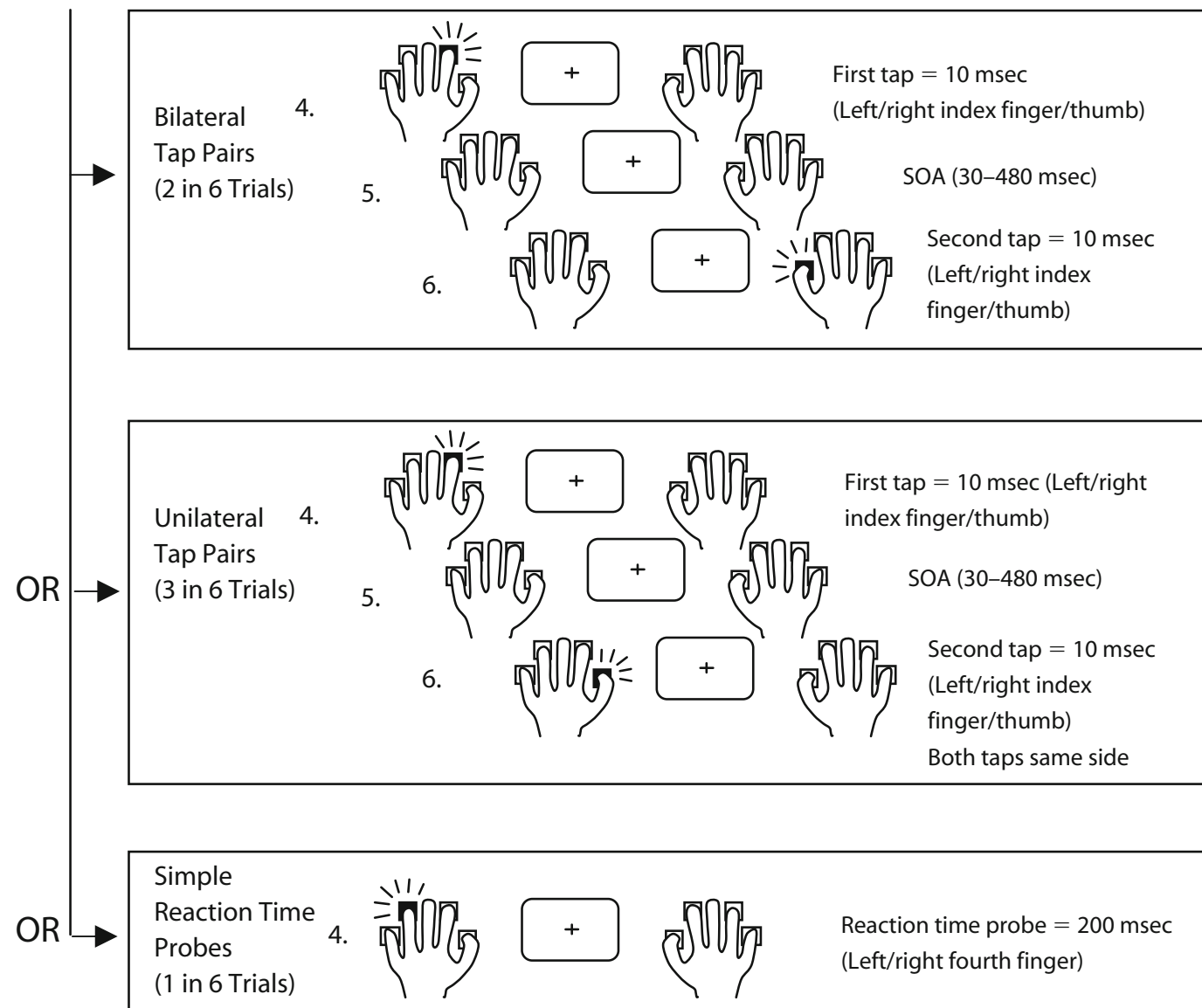

Figure 2. Sample sequence of events for a single trial in the exogenous cuing experiment (Experiment 1). The diagram is schematic only and does not represent the actual posture of the hands during the experiment. For exogenous cuing, the central fixation cross was displayed until a response was made. For endogenous cuing, precue central fixation cross display time was also $300 \mathrm{msec}$, followed by display of the cue-a centrally presented arrow pointing left, right, or in both directions. The cue was at the same location as the fixation cross. The cue display time for endogenous cuing was $\mathbf{4 0 0}$ msec. Only data from bilateral trials were relevant to the experimental hypotheses. Unilateral trials were included to induce participants to direct their attention in the direction of the arrow cues in the endogenous cuing experiment. In this experiment, all unilateral tap pairs were presented on the side to which the cuing arrow pointed. 
Bilateral temporal order judgment trials. In two out of six trials, $80 \mathrm{msec}$ after the cue, two temporal order taps were administeredone tap to each hand - separated by a variable interval. The taps were always presented in pairs of different elevations - that is, one tap to the index finger (upper elevation) of one hand and one tap to the thumb (lower elevation) of the other hand. One tap was presented to the left hand and one to the right hand for all trials. In half of the trials, a digit on the right hand (index finger or thumb) was stimulated first, and in the remaining trials, a digit on the left hand was stimulated first. The taps were separated by 1 of 10 randomly assigned intervals ranging from $-480 \mathrm{msec}$ to $+480 \mathrm{msec}(-480,-270,-180$, $-90,-30,+30,+90,+180,+270,+480 \mathrm{msec})$. The cue duration of $20 \mathrm{msec}$, plus an $80-\mathrm{msec}$ interval prior to the first experimental tap $-100 \mathrm{msec}$ in total from the cue onset to first stimulus onsetwas chosen to closely approximate the total duration separating cue onset from the first stimulus onset in the Shore et al. (2001) study. In that study, the exogenous cue was displayed for $45 \mathrm{msec}$, followed by a $60-\mathrm{msec}$ interval to the first experimental stimulus.

Participants made a temporal order judgment about the two taps. Half of the participants were instructed to make unspeeded "which first?" judgments - that is, "Was the first tap an upper/index finger tap or lower/thumb tap?" The remaining half made the converse "which second?" judgments. The side to which the taps were delivered was irrelevant to the task. The manipulation of spatial attention (left-right, horizontal dimension) was therefore orthogonal to the response dimension (upper-lower, vertical dimension). Participants responded in a forced choice manner by releasing one of two pedals located under the toe and heel of the right foot. Release of the toe pedal indicated that the upper tap was judged first (or second, depending on task instructions). Release of the heel pedal indicated that the lower tap was judged first (or second, depending on the task). This mapping of pedal responses to tap elevations was kept constant throughout the experiment and was the most spatially intuitive, since participants perceived the toe pedal as the "upper" pedal and the heel pedal as the "lower" pedal.

Unilateral temporal order judgment trials. In three out of six trials, $80 \mathrm{msec}$ after the cue, two temporal order taps were presented (one upper and one lower tap). These taps were both on the same hand, half of the time on the same side as the cue, the other half of the time on the opposite side of the cue, and with equal probability on the left and right hands when the cue was applied to both hands. This condition was necessary for reasons relating to endogenous manipulation of attention in Experiment 2. These trials were effectively "dummy" trials.

Reaction time probe trials. In one out of six trials, $80 \mathrm{msec}$ after the cue, reaction time probes were delivered to the ring finger of either the left or the right hand. All participants were instructed to respond to this stimulus by releasing the pedal located under their left foot as fast as possible, irrespective of the hand to which the stimulus was delivered. Reaction time was recorded.

General information. Participants were told to focus on accuracy with no instruction to give rapid responses, unless responding to a reaction time probe. The intertrial interval was $1,500 \mathrm{msec}$. Feedback regarding accuracy was given at the conclusion of each experimental block.

Participants completed six blocks of 120 trials each. A practice block of 72 trials was delivered prior to testing. In this block, relatively large stimulus onset asynchronies (SOAs) $(180 \mathrm{msec}$ and $270 \mathrm{msec}$ ) between the two taps were used to facilitate learning of the task. Feedback was given after every trial in the practice block. The practice block was repeated until a performance hurdle of $70 \%$ correct was achieved.

\section{Results and Discussion}

Effect of attention cues on simple reaction time task. Mean reaction times to the intermittently presented tactile probe stimuli were analyzed with a two-factor mixed model ANOVA. Cue validity was the within-participants

\section{Exogenous Cuing}

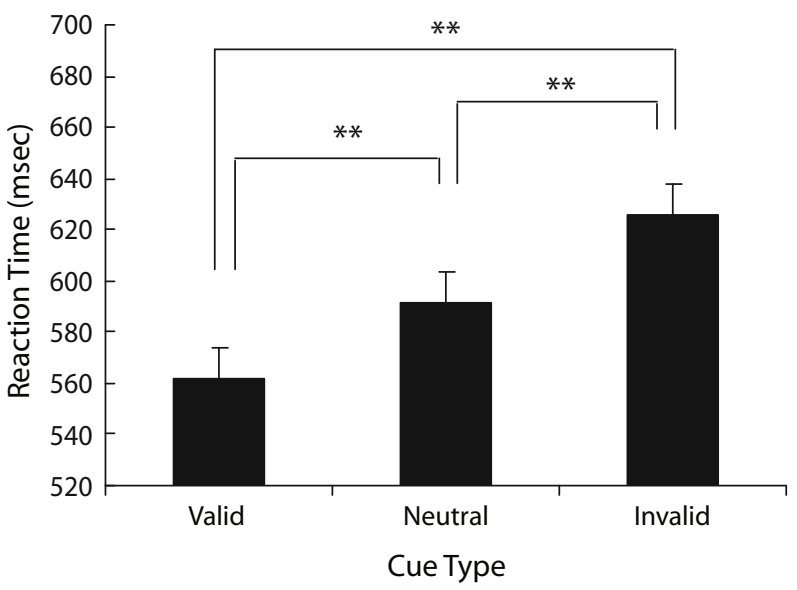

Figure 3. The effect of cue type on reaction times to intermittent probe stimuli-exogenous cues (valid, cue and probe on same side; neutral, cues on both sides; invalid, cue and probe on opposite sides). Error bars represent root mean square error, recommended for within-participants designs (Estes, 1997). ${ }^{* *} p<.01$ (two-tailed $t$ tests).

factor, with three levels: valid, neutral, and invalid. Task type was the between-participants factor, with two levels: "which first" and "which second," referring to the instructions that the participants received for the temporal order judgment task, either "which tap first?" or "which tap second?" The slowest $10 \%$ of reaction times were removed prior to analysis. Effect sizes are expressed as $\eta_{\mathrm{p}}^{2}$ values.

Figure 3 shows a significant main effect of cue validity $\left[F(2,16)=71.981, p<.001, \eta_{\mathrm{p}}^{2}=.9, p w=1.0\right]$ with reaction times to valid trials being faster than those to neutral trials, which in turn were faster than those to invalid trials. Paired-samples $t$ tests (two-tailed) between valid and invalid, valid and neutral, and neutral and invalid trials were all significant (see Figure 3 ). There was no main effect of task type $\left[F(1,8)=1.63, p>.1, \eta_{\mathrm{p}}^{2}=\right.$ $.169, p w=.204]$, and no interaction between the factors $[F(2,16)<1]$. These results demonstrate that spatial attention was manipulated effectively by the exogenous cuing used in this experiment, consistent with results reported by Shore et al. (2001), who studied prior entry within vision, and with results reported more widely (Posner, 1988).

Effect of attention cues on temporal order judgments. For each participant, the proportion of "upper tap first" responses was plotted as a function of the SOA between the taps for the three cuing conditions (lower tap cued, upper tap cued, or neutral-both taps cued). Averaged data are shown in Figure 4. Cumulative normal distributions were fitted to the plotted responses; these smoothed functions captured the participants' entire response distributions. These were used to estimate the points of subjective simultaneity (PSS) for each of the three cuing conditions (derived as the SOA at which the smoothed response distributions for the three cuing conditions crossed .5 on the $y$-axis-i.e., the point at which participants responded 

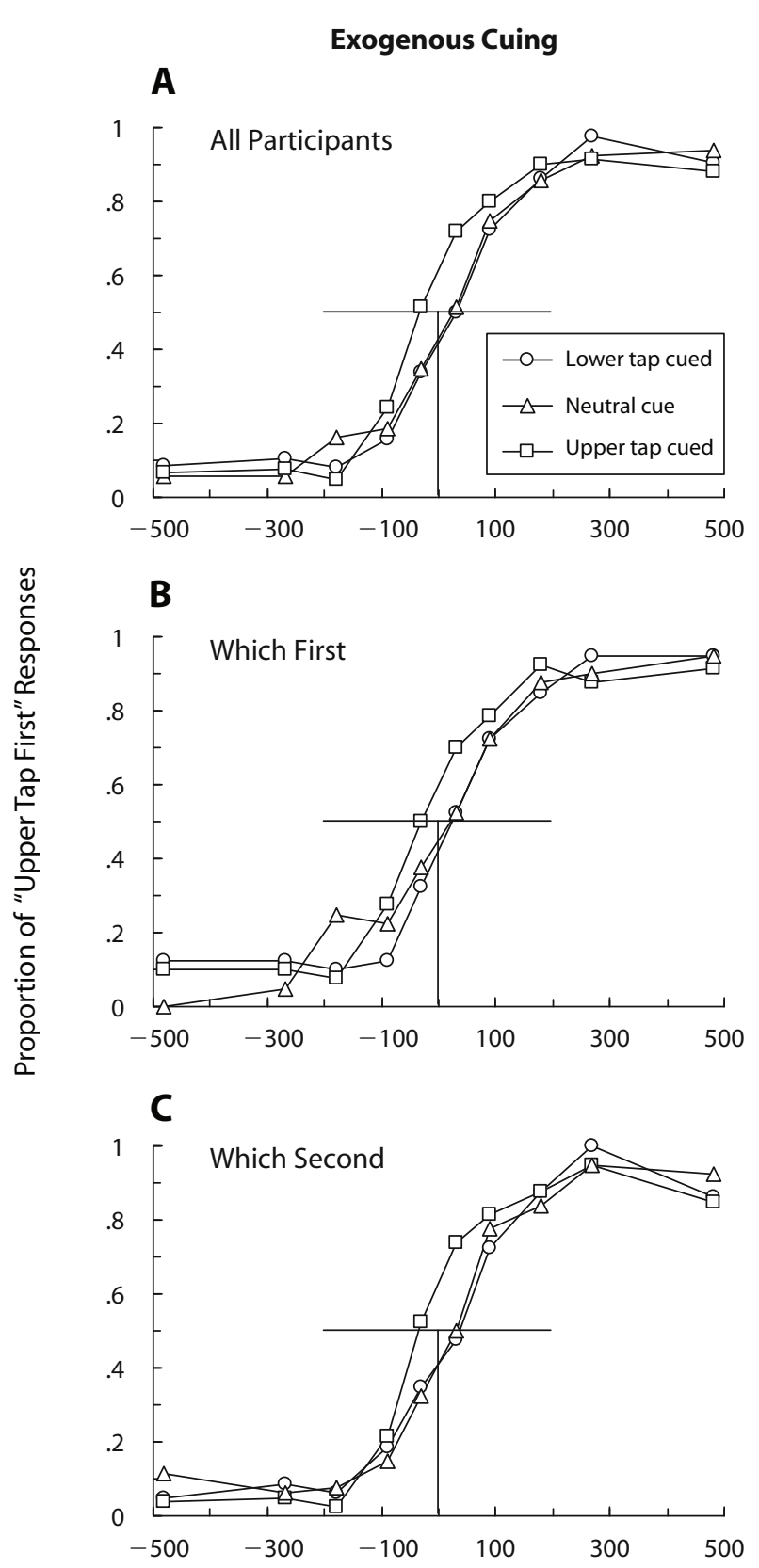

Lower Tap First SOA (msec) Upper Tap First

Figure 4. Temporal order judgment data, exogenous cues. (A) Average data for all participants. (B) Average data for participants instructed to judge "which tap first?" (C) Average data for participants instructed to judge "which tap second?"

"upper tap first" and "lower tap first" equally often). This method of interpolating the PSS for each cuing condition using the participants' entire response distributions represents an improvement on previous methods (see, e.g., Shore et al., 2001) in which the PSS was derived through linear interpolation of only the two data points immediately above and below .5. With this new method, the derived PSS values are less susceptible to significant distortion as a consequence of a single anomalous data point. An assessment of goodness-of-fit was conducted on the resulting 30 individual psychometric functions (10 participants $\times 3$ cuing conditions). Kolmogorov-Smirnov $D$ statistic values (in Stephens, 1986) for these functions ranged from 0.23 to 1.66 , with a mean of 0.79 . Only 3 out of the 30 functions $(10 \%)$ were not a good fit for the observed data, using an $\alpha$ significance level of 05 .

When the lower/thumb taps were cued (cue delivered to the same side as the lower tap), the mean PSS, averaged across task type, was $+26 \mathrm{msec}$ (positive value is by arbitrary convention and indicates that the PSS occurred when the upper tap preceded the lower tap by $26 \mathrm{msec}$, implying that cuing the lower tap accelerated the perception of lower taps). The mean PSS when the upper/index finger taps were cued was $-22 \mathrm{msec}$ (i.e., when the lower tap preceded the upper tap by $22 \mathrm{msec}$ ). The mean PSS when both taps were cued was +13 msec. The mean PSS values for all conditions for Experiment 1 (and Experiment 2), including mean PSS values by task type, are presented in their clearest form in Table A1 in the Appendix. PSS values were analyzed using a two-factor mixed-model ANOVA. Cue condition was the within-participants factor, with three levels (lower tap cued, upper tap cued, or neutral: both lower and upper taps cued). Task type was the between-participants factor with two levels: "which first" and "which second."

There was a significant main effect of cue condition $\left[F(2,16)=6.201, p<.05, \eta_{\mathrm{p}}^{2}=.437, p w=.824\right]$, indicating that directing attention to a particular location using exogenous cues had a significant effect on the point at which participants judged two subsequent taps (one cued and one not cued) as simultaneous. The difference in the PSS for the lower-tap-cued and upper-tap-cued conditions was $48 \mathrm{msec}$. A paired samples $t$ test (two-tailed) conducted on the lower-tap-cued and upper-tap-cued PSSs was significant $[t(9)=4.235, p<.01]$. There was no main effect of task type $[F(1,8)<1]$, and no interaction between the factors was observed $[F(2,16)<1]$.

Last, an averaged PSS for each participant (averaged across cuing conditions) was calculated and analyzed with a one-sample $t$ test (two-tailed) to determine whether there was any systematic tendency to perceive the taps as simultaneous at a point in time other than the point at which they were actually simultaneous, regardless of the cuing condition. No such effect was observed; the grand mean PSS was $+6 \operatorname{msec}[t(9)=0.512, p>.5]$.

These results support the existence of a robust prior entry effect that is present across different sensory modalities (cf. Shore et al., 2001) when spatial attention is manipulated exogenously. The magnitude of the effect for the tactile modality is calculated as the average shift in PSS across both the lower- and upper-tap-cued conditions, equivalent to half the magnitude of the interval between the mean PSS for the lower- and upper-tap-cued conditions. This yields an estimated prior entry effect of $24 \mathrm{msec}$. By comparison, the magnitude of prior entry within vision, using exogenous cuing of attention, was estimated to be $61 \mathrm{msec}$ (Shore et al., 2001). 


\section{EXPERIMENT 2 Endogenous Cuing}

In Experiment 2, we investigated the influence of endogenously generated shifts of attention on the perceived timing of somatosensory stimuli. By addressing exogenous and endogenous cuing of attention separately, the impact of the each cuing type can be distinguished. Endogenous cuing is also useful in overcoming difficulties in investigating prior entry that are related specifically to exogenous cuing. In exogenous cuing, an observed perceptual acceleration for attended stimuli might result from sensory/perceptual factors other than attention. Alternative interpretations are sensory facilitation and temporal attraction between the spatially proximate cue and target stimuli (Schneider \& Bavelier, 2003). The investigation of prior entry using endogenously manipulated attention, which uses symbolic cues, neatly sidesteps these potential confounds.

\section{Method}

Participants. Twenty-two healthy students took part in this experiment in return for course credit at the University of Melbourne. The complete data set (reaction time and temporal order judgment data) was removed for 1 participant after it was discovered that white noise delivery through the headphones had failed midway through the experiment. All remaining participants ( 9 men, 12 women) except 1 were strongly right-handed.

Apparatus and Stimuli. The apparatus and stimuli were the same as those used for Experiment 1.

Procedure. The procedure was the same as that in Experiment 1 , except attention was manipulated endogenously. Centrally presented arrows pointed left, right, or in both directions (doubleheaded), directing participants to attend to the left or right hands or to divide their attention across both hands. The different arrow cues were equiprobable, randomly selected, and displayed for $400 \mathrm{msec}$. Arrow cues were followed by the two experimental taps (10-msec duration), one tap to each hand, one upper and one lower tap, as per Experiment 1, and task conditions were also equivalent to those in Experiment 1. The cue display time of $400 \mathrm{msec}$ was chosen to closely approximate the endogenous cue display time in the Shore et al. (2001) article to maximize the equivalence between that study and the present study. As with that study, the arrow cues were spatially predictive. In $75 \%$ of trials in the present study, the first experimental tap after the cue appeared on the side to which the arrow pointed, in order to encourage endogenous shifts of attention in the direction of the arrow. Achieving this ratio necessitated the creation of dummy trials ( 360 trials, equivalent in number to three blocks out of a total of six blocks) interspersed across all blocks in which both upper and lower taps occurred on the side to which the cue arrow pointed. Only trials in which the two taps were presented one each to the two hands were used to test the experimental hypothesis. In Experiment 1, dummy trials were also included with upper and lower taps on the same side, but with these same-side tap pairs presented equally on the cued and noncued sides so that exogenous cues were spatially uninformative. Doing this was necessary to make the exogenous and endogenous manipulations of attention as comparable as possible.

\section{Results and Discussion}

Effect of attention cues on simple reaction time task. Mean reaction times to the intermittently presented tactile probe stimuli were analyzed with a two-factor (cue validity and task type) mixed-model ANOVA, as was the

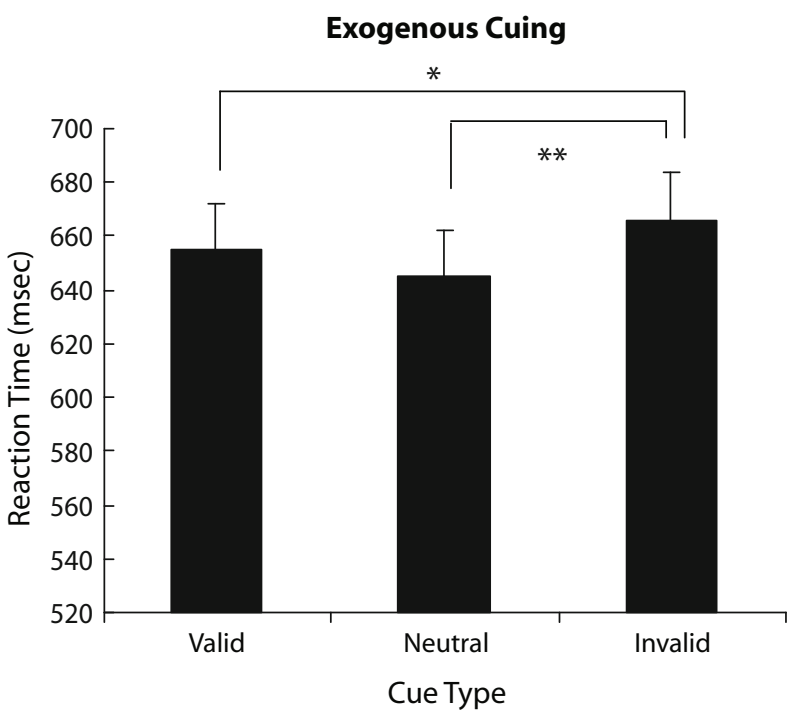

Figure 5. Effect of cue type on reaction times to intermittent probe stimuli-endogenous cues (valid, cue arrow in direction of probe; neutral, bidirectional cue arrow; invalid, cue arrow in opposite direction to probe). Error bars represent root mean square errors, recommended for within-participants designs (Estes, 1997). ${ }^{*} p<.05 .{ }^{* *} p<.01$ (two-tailed $t$ tests).

case for Experiment 1 . The slowest $10 \%$ of reaction times were removed prior to analysis.

Figure 5 shows a significant main effect of cue validity $\left[F(2,38)=7.354, p<.005, \eta_{\mathrm{p}}^{2}=.279, p w=.92\right]$ on mean reaction times to the tactile probe stimuli. Paired-samples $t$ tests (two-tailed) revealed that reaction times to validly cued trials and to neutral trials were both significantly faster than those to invalidly cued trials (see Figure 5). There was no main effect of task type $[F(1,19)=4.168$, $\left.p>.05, \eta_{\mathrm{p}}^{2}=.18, p w=.491\right]$ and no interaction between cue validity and task type $\left[F(2,38)=1.696, p>.1, \eta_{\mathrm{p}}^{2}=\right.$ $.082, p w=.33]$.

Effect of attention cues on temporal order judgments. For each participant, the proportion of "upper tap first" responses was plotted as a function of the SOA between the taps for the three cuing conditions. Averaged data are shown in Figure 6. PSS values were derived in the same manner as for Experiment 1. An assessment of goodness-of-fit was conducted on the resulting 63 individual psychometric functions (21 participants $\times 3$ cuing conditions). Kolmogorov-Smirnov $D$ statistic values (Stephens, 1986) for these functions ranged from 0.19 to 1.40 , with a mean of 0.62 . Only 1 out of the 63 functions $(1.6 \%)$ was not a good fit for the observed, data using an $\alpha$ significance level of 05 .

The mean PSS, averaged across task type, when lower taps were cued (arrow pointed to the side at which the lower tap was delivered) occurred at $-9 \mathrm{msec}$ - that is, when the lower tap preceded the upper tap by 9 msec. When upper taps were cued, the mean PSS was $-42 \mathrm{msec}$. When both taps were cued, the mean PSS was -23 msec. Refer to Table A1 in the Appendix for the full set of mean PSS values. The data were analyzed using the same two- 


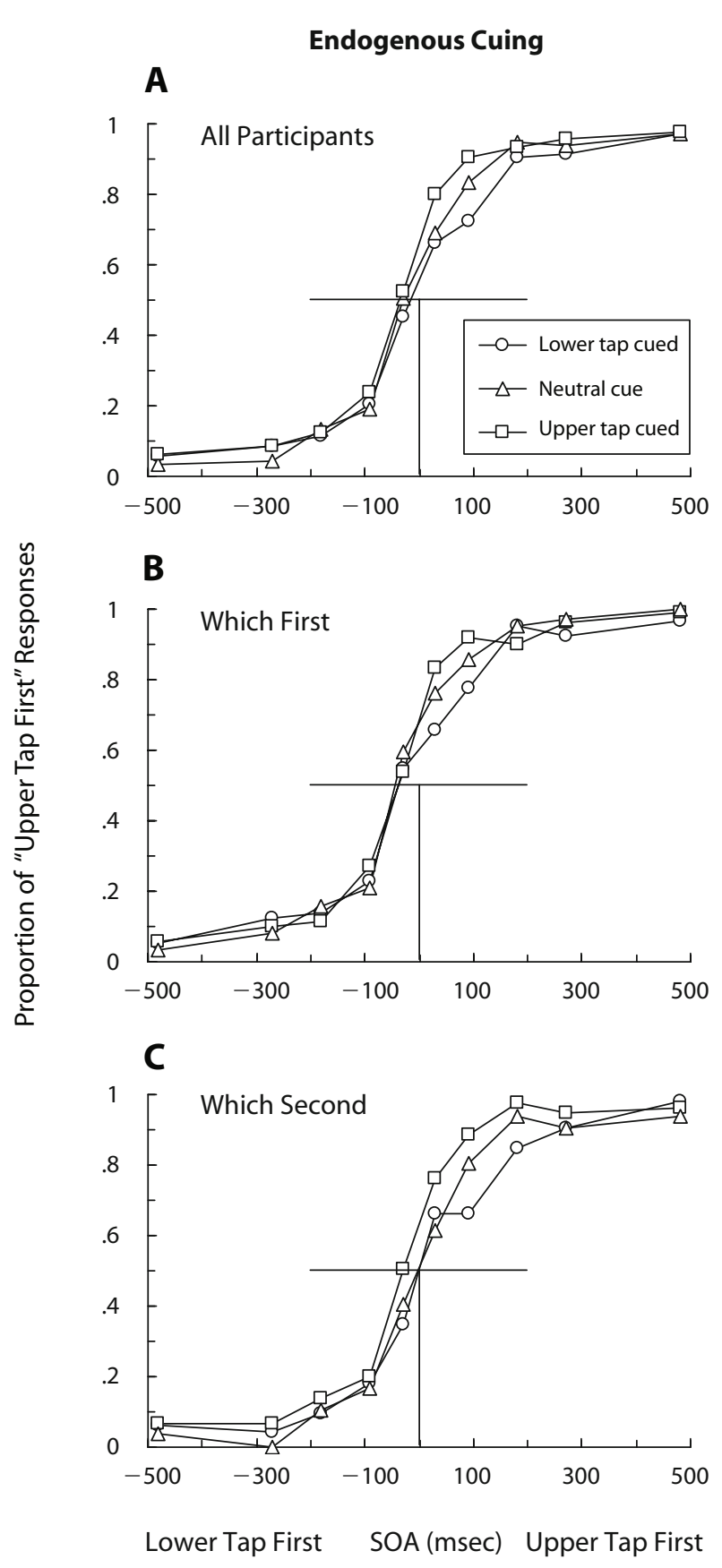

Figure 6. Temporal order judgment data, endogenous cues. (A) Average data for all participants. (B) Average data for participants instructed to judge "which tap first?" (C) Average data for participants instructed to judge "which tap second?"

factor mixed-model ANOVA (cue condition $\times$ task type) as in Experiment 1.

There was a significant main effect of cue condition $\left[F(2,36)=5.285, p<.01, \eta_{\mathrm{p}}^{2}=.218, p w=.805\right]$, indicating that directing attention to a particular location using endogenous cues had a significant effect on the SOA at which participants judged two subsequent taps (one cued and one not cued) as simultaneous. The difference between
PSSs for the lower tap cued and upper tap cued conditions was $33 \mathrm{msec}$. A paired samples $t$ test (two-tailed) of the lower-tap-cued and upper-tap-cued PSSs was significant $[t(20)=2.94, p<.01]$, indicating that the prior entry effect for endogenously manipulated attention has an estimated magnitude of $16.5 \mathrm{msec}$ (half of the mean PSS interval of the nonneutral cue conditions; see Experiment 1 Results section for this derivation).

Unlike in Experiment 1, there was a main effect of task type when attention was manipulated endogenously $\left[F(1,19)=5.120, p<.05, \eta_{\mathrm{p}}^{2}=.212, p w=.647\right]$. The average PSS, regardless of cuing condition, for the "which first" condition was $-46 \mathrm{msec}$, and the equivalent PSS for the "which second" condition was -2 msec. A bias toward perceiving upper taps earlier than lower taps specific to the "which first" condition could account for this negative/leftward shift of the PSS in that condition. Possible explanations of such a bias are considered in the General Discussion section. There was no interaction between cue condition and task type $[F(2,38)<1]$.

As was done for Experiment 1, an average PSS was calculated for each participant (averaged across cuing conditions) to establish whether participants exhibited a systematic bias to perceive the taps as simultaneous at a point in time other than when they were actually simultaneous, irrespective of cuing condition. These averaged PSS values were analyzed with a one-sample $t$ test (twotailed) that revealed a significant negative/leftward deviation of PSS values from true simultaneity; the grand mean PSS was $-25 \operatorname{msec}[t(20)=-2.343, p<.05]$. This result is principally due to a large negative/leftward shift in average PSS values for the "which first" condition, and is considered in context with the significant main effect of task type in the General Discussion.

\section{GENERAL DISCUSSION}

The present study provides support for a prior entry effect operating within the somatosensory modality. The reduced perceptual latency for attended stimuli as compared with unattended stimuli was found both when the focus of spatial attention was externally manipulated (exogenous cuing) and when it was internally generated by the participant (endogenous cuing), with a more marked effect for externally manipulated attention. In contrast with much of the previous work investigating prior entry, the present study used an experimental procedure specifically designed to minimize the role of response bias. This is the first such study addressing within-modality prior entry for somatosensory stimuli.

The prior entry effect found in the present study is consistent with earlier related studies that examined the effect of attention on processing speed in the somatosensory modality. These showed more rapid tactile discrimination responses for cued versus uncued targets, both exogenously (Spence \& McGlone, 2001) and endogenously (Spence, Pavani, \& Driver, 2000). The more direct comparison of the present study with previous work on prior entry within vision (Shore et al., 2001) also reveals some similarities in 
the nature of the effect across different sensory modalities. First, in both modalities, the magnitude of prior entry was greater when shifts in attention were elicited by exogenous cues. Second, estimates of the magnitude of prior entry for the endogenous cuing condition were very similar across both modalities - around $17 \mathrm{msec}$. Third, in both studies, the size of the prior entry effect observed within each modality was proportional to the magnitude of reaction time facilitation in an embedded task comparing reaction times to probes at cued and noncued locations. These similarities suggest the possibility of the existence of a common modality-independent mechanism that mediated the effects observed for the two modalities. This mechanism may have been attention-driven latency reductions and/ or magnitude increases of sensory neuronal responses. The latest possible operation (i.e., upper temporal limit) of such a mechanism is just prior to the point at which the sensory signals are converted into perceptual events with subjective temporal properties; any later than this and no effect on perceived timing would be observed. The earliest possible operation (i.e., lower temporal limit) is more difficult to place. If the neural mechanism of prior entry was modality independent, this would suggest that the mechanism operated subsequent to the integration of sensory signals from different modalities, which is known to occur to some extent as early as the superior colliculus (Meredith \& Stein, 1986).

\section{Reaction Time Probe Task}

The use of a reaction time task incorporated into the experimental design made it possible to gauge the effectiveness of the cuing of spatial attention independently of any prior entry effect, and the significant reaction time advantage for the cued locations for both types of cuing provided confirmation of its effectiveness. In both the present study and the Shore et al. (2001) visual prior entry study, reaction time facilitation was greater in the exogenous condition than in the endogenous condition-64 to $11 \mathrm{msec}$ in the present study and 38 to $23 \mathrm{msec}$ for Shore et al. (2001). Consequently, the greater magnitude of the observed prior entry effect for exogenous cuing for both modalities may have resulted from a more substantial, or more complete, shift in the location of attention resulting from the manipulation of attention by exogenous as opposed to endogenous cuing.

\section{Response Bias}

By using orthogonal cuing and response dimensions, the present study avoided the most obvious potential form of response bias - that is, the bias to make a "left" response when the left side was cued, and its converse for the right side, but it could not directly circumvent secondorder response bias, as was described earlier. The use of two tasks- -"which first" and "which second"-offered a means to address this particular form of bias. Any secondorder response bias should manifest as an increased separation of PSS values in the different cuing conditions for the first task, and as a decreased separation in the second task, observable as an interaction between cue condition and task type. In neither exogenous nor endogenous manipulations of attention did this interaction achieve statistical significance.

\section{Effect of Task Type and Perceptual Biases}

An additional feature of the results, which does not affect the central finding of this study of attention-induced acceleration of perceived timing, but which is nevertheless interesting, is the effect of task type in Experiment 2. A significant leftward/negative shift in the average PSS values was observed for the "which first" condition. This led to a significant overall leftward shift of PSS values regardless of the task condition. A tendency toward perceiving upper taps earlier than lower taps could account for the observed leftward shift of PSS values for the "which first" condition. A possible explanation for this is that when cuing was endogenous, the resulting shifts of attention were lateralized but also biased upward. Such a tendency is possible, given both known biases to preferentially attend the upper part of the visual field (McCourt \& Olafson, 1997) and observations of more rapid responses to upper versus lower tactile and visual targets in a study investigating cross-modal links in endogenous spatial attention (Spence et al., 2000). An attentional focus above the taps could have resulted in earlier perception of the closer, upper taps as compared with the more distant, lower taps. Endogenous shifts of attention might be expected to be more susceptible to mislocalization and bias than are exogenous shifts of attention, for which the peripheral cue serves as a location anchor. This could explain why there was no bias to perceiving upper taps first during exogenous cuing. A possible explanation for the leftward shift in PSS values occurring only for the "which first" task condition in the endogenous cuing experiment is the different temporal allocation of attention in the two tasks, and how this might interact with the process of shifting spatial attention. Any mislocalization tendency in the allocation of spatial attention may be particularly pronounced if there is a competing concurrent task-for example, allocation of attention on the temporal dimension, which might be expected to be greater in the "which first" task, which is earlier in time and therefore more likely to coincide with the active shifting of spatial attention.

Also of interest was the greater proximity of the neutral mean to the lower-tap-cued mean than to the upper-tapcued mean in the exogenous cuing experiment. The mean of the PSS values for the neutral condition was not significantly different from true simultaneity, but the effect is suggestive of greater perceptual latency reductions for cued upper taps as compared with cued lower taps.

\section{Eye Movements}

For both experiments, we were interested in the effects of "pure" covert shifts in spatial attention on the perceived timing of subsequently presented stimuli, dissociated from overt orienting, for example saccades (Corbetta, 1998) or head turns. In the present study, head position was fixed and participants were instructed to 
maintain fixation on a centrally presented cross throughout the trial. Because eye movements were not monitored, we cannot rule out the possibility of saccades made in response to the tactile cues. This possibility could be investigated in future research, and any potential impact of such saccades on perceived timing could be evaluated. However, it is worth noting that - at least in the exogenous cuing condition - the accelerated perception of targets on the cued side was unlikely to be due to saccades, given that the time interval between the cue and first target was less than the time required to initiate a saccade (Schneider \& Bavelier, 2003).

\section{Future Directions and Research}

The present study provides evidence in support of prior entry in the somatosensory modality, supporting the view that prior entry is a general phenomenon of our sensory apparatus. The demonstration of the effect for somatosensory stimuli indicates that prior entry is not dependent on any particular range of latencies with which sensory signals from each modality reach the primary sensory cortex, given the divergent neural latencies of visual and somatosensory afferent signals. Future work on prior entry within audition would deliver a more complete picture of the phenomenon.

Within somatosensation, knowledge of the spatial parameters of the prior entry effect could be greatly sharpened. Currently, it is not known whether stimuli are perceived more rapidly if they are located within an entire attended hemifield/side of the body's midline, or whether they must be located within a more confined attended region. Nor is it known whether the effect operates in body-centered or environment-centered spatial coordinates. Simple postural manipulations would be able to address this issue and, in so doing, help locate the neural basis of the effect. Additionally, as was mentioned earlier, monitoring of eye movements during attentional cuing will tease out the contributions of overt versus covert location of attention to the prior entry effect.

A significant recent development has been the use of electrophysiological measures to investigate the neural underpinnings and time course of prior entry. At this point, there are conflicting results (McDonald et al., 2005; Vibell, Klinge, Zampini, Spence, \& Nobre, 2007). The application of these techniques to the study of prior entry within touch presents a useful new line of evidence that can be recruited to help resolve these discrepancies. Further insight into the neural underpinnings of prior entry could be gleaned from transcranial magnetic stimulation studies exploring the impact of time-specific and sitespecific neural disruption on the prior entry effect.

\section{AUTHOR NOTE}

The authors thank Jason Mattingley for assistance with experimental design and Max Rademacher for technical support. We gratefully acknowledge the support of Australian Research Council Grant DP0557576 to M.E.R.N. M.J.Y. is also supported by an Australian Postgraduate Award. Address correspondence to M. J. Yates, Department of Psychology, University of Melbourne, Parkville, VIC 3010, Australia (e-mail: mjyates@unimelb.edu.au).

\section{REFERENCES}

Bertelson, P., \& Aschersleben, G. (2003). Temporal ventriloquism: Cross-modal interaction on the time dimension: 1. Evidence from auditory-visual temporal order judgment. International Journal of Psychophysiology, 50, 147-155.

CAIRnEY, P. T. (1975). Bisensory order judgment and prior entry hypothesis. Acta Psychologica, 39, 329-340.

CorbetTA, M. (1998). Frontoparietal cortical networks for directing attention and the eye to visual locations: Identical, independent, or overlapping neural systems? Proceedings of the National Academy of Sciences, 95, 831-838.

Coull, J. T., \& Nobre, A. C. (1998). Where and when to pay attention: The neural systems for directing attention to spatial locations and to time intervals as revealed by both PET and fMRI. Journal of Neuroscience, 18, 7426-7435.

Cunningham, D. W., Billock, V. A., \& Tsou, B. H. (2001). Sensorimotor adaptation to violations of temporal contiguity. Psychological Science, 12, 532-535.

Downar, J., Crawley, A. P., Mikulis, D. J., \& Davis, K. D. (2000). A multimodal cortical network for the detection of changes in the sensory environment. Nature Neuroscience, 3, 277-283.

Ehrsson, H. H., Spence, C., \& Passingham, R. E. (2004). That's my hand! Activity in premotor cortex reflects feeling of ownership of a limb. Science, 305, 875-877.

EsTES, W. K. (1997). On the communication of information by displays of standard errors and confidence intervals. Psychonomic Bulletin \& Review, 4, 330-341.

Friedrich, F. J., Egly, R., Rafal, R. D., \& Beck, D. (1998). Spatial attention deficits in humans: A comparison of superior parietal and temporal-parietal junction lesions. Neuropsychology, 12, 193-207.

Funes, M. J., Lupí́ñez, J., \& Milliken, B. (2007). Separate mechanisms recruited by exogenous and endogenous spatial cues: Evidence from a spatial Stroop paradigm. Journal of Experimental Psychology: Human Perception \& Performance, 33, 348-362.

Hein, E., Rolke, B., \& UlRich, R. (2006). Visual attention and temporal discrimination: Differential effects of automatic and voluntary cuing. Visual Cognition, 13, 29-50.

HikosaKa, O., MiYauchi, S., \& Shimojo, S. (1993). Focal visual attention produces illusory temporal order and motion sensation. Vision Research, 33, 1219-1240.

JASKOWSKI, P. (1993). Selective attention and temporal-order judgment. Perception, 22, 681-689.

Kincade, J. M., Abrams, R. A., Astafiev, S. V., Shulman, G. L., \& Corbetta, M. (2005). An event-related functional magnetic resonance imaging study of voluntary and stimulus-driven orienting of attention. Journal of Neuroscience, 25, 4593-4604.

McCourt, M. E., \& Olafson, C. (1997). Cognitive and perceptual influences on visual line bisection: Psychophysical and chronometric analyses of pseudoneglect. Neuropsychologia, 35, 369-380.

McDonald, J. J., Teder-Sälejärvi, W. A., Di Russo, F., \& Hillyard, S. A. (2005). Neural basis of auditory-induced shifts in visual timeorder perception. Nature Neuroscience, 8, 1197-1202.

Meredith, M. A., \& Stein, B. E. (1986). Visual, auditory, and somatosensory convergence on cells in superior colliculus results in multisensory integration. Journal of Neurophysiology, 56, 640-662.

Miura, T., Sonoo, M., \& Shimizu, T. (2003). Establishment of standard values for the latency, interval and amplitude parameters of tibial nerve somatosensory evoked potentials (SEPs). Clinical Neurophysiology, 114, 1367-1378.

OLDFIELD, R. C. (1971). The assessment and analysis of handedness: Edinburgh inventory. Neuropsychologia, 9, 97-113.

PAshler, H. E. (1998). The psychology of attention. Cambridge, MA: MIT Press.

Posner, M. I. (1988). Structures and functions of selective attention. In T. Boll \& B. Bryant (Eds.), Master lectures in clinical neuropsychology (pp. 173-202). Washington, DC: American Psychological Association.

Posner, M. I., \& Petersen, S. E. (1990). The attention system of the human brain. Annual Review of Neuroscience, 13, 25-42.

Rorden, C., Mattingley, J. B., Karnath, H. O., \& Driver, J. (1997). Visual extinction and prior entry: Impaired perception of temporal 
order with intact motion perception after unilateral parietal damage. Neuropsychologia, 35, 421-433.

SCHNEIDER, K. A., \& BAVELIER, D. (2003). Components of visual prior entry. Cognitive Psychology, 47, 333-366.

Shore, D., \& Spence, C. (2005). Prior entry. In L. Itti, G. Rees, \& J. Tsotsos (Eds.), Neurobiology of attention (pp. 89-95). Amsterdam: Elsevier.

Shore, D., Spence, C., \& Klein, R. M. (2001). Visual prior entry. Psychological Science, 12, 205-212.

SPENCE, C., \& Driver, J. (2004). Cross-modal space and cross-modal attention. Oxford: Oxford University Press.

Spence, C., \& McGlone, F. P. (2001). Reflexive spatial orienting of tactile attention. Experimental Brain Research, 141, 324-330.

Spence, C., Pavani, F., \& Driver, J. (2000). Cross-modal links between vision and touch in covert endogenous spatial attention. Journal of Experimental Psychology: Human Perception \& Performance, 26, 1298-1319.

Spence, C., Shore, D. I., \& Klein, R. M. (2001). Multisensory prior entry. Journal of Experimental Psychology: General, 130, 799-832.

Stelmach, L. B., \& Herdman, C. M. (1991). Directed attention and perception of temporal order. Journal of Experimental Psychology: Human Perception \& Performance, 17, 539-550.

StEPHENS, M. (1986). Tests based on EDF statistics. In R. D'Agostino \& M. Stephens (Eds.), Goodness-of-fit techniques. New York: Dekker.
TiTCHENER, E. B. (1908). Lectures on the elementary psychology of feeling and attention. New York: Macmillan.

VANDERhaeghen, C., \& BerTelson, P. (1974). The limits of prior entry: Nonsensitivity of temporal order judgments to selective preparation affecting choice reaction time. Bulletin of the Psychonomic Society, 4, 569-572.

Vibell, J., Klinge, C., Zampini, M., Spence, C., \& Nobre, A. C. (2007). Temporal order is coded temporally in the brain: Early eventrelated potential latency shifts underlying prior entry in a cross-modal temporal order judgment task. Journal of Cognitive Neuroscience, 19, 109-120.

Wegner, D. M., \& Wheatley, T. (1999). Apparent mental causation: Sources of the experience of will. American Psychologist, 54, 480-492.

Yamada, K., Kaga, K., Uno, A., \& Shindo, M. (1996). Sound lateralization in patients with lesions including the auditory cortex: Comparison of interaural time difference (ITD) discrimination and interaural intensity difference (IID) discrimination. Hearing Research, 101, 173-180.

Zampini, M., Bird, K. S., Bentley, D. E., Watson, A., Barrett, G., Jones, A. K., \& SPEnce, C. (2007). "Prior entry" for pain: Attention speeds the perceptual processing of painful stimuli. Neuroscience Letters, 414, 75-79.

Zampini, M., Shore, D. I., \& Spence, C. (2005). Audiovisual prior entry. Neuroscience Letters, 381, 217-222.

\section{APPENDIX}

The averaged graphed data (in Figures 4 and 6) yield SOAs at which each cuing function intercepts .5 on the $y$-axis (apparent average PSS values). These values are discrepant from the values in Table A1. This is a consequence of how the averaged data are generated in the different formats. In the graphed format, for each pairing of cuing condition and SOA, a data point is derived that is the average of all participants - which is, in effect, an average of a range of vertically dispersed values (different $y$-axis values) given a fixed horizontal value (the specified SOA). The point at which each averaged response function crosses the .5 level on the $y$-axis is then a consequence of the distance from this .5 level of the average values for the SOAs that bound it above and below. The mathematically derived PSS values used for the statistical analysis, which are presented as average PSS values in Table A1, were calculated by fitting cumulative normal distributions to the response functions for each participant and computing the intercepts with the .5 level on the $y$-axis, yielding a horizontal dispersion of values (different SOA intercepts of .5 on the $y$-axis). This horizontal dispersion of values for each cuing condition was then averaged to calculate the average PSS for that cuing condition. It would be possible to graph the response functions in such a way that the average PSS values derived graphically for each cuing condition precisely matched the average PSS values derived mathematically. This could be done by using the cumulative normal functions fitted to each participant's data to derive the SOAs at which specified $y$-axis values are intercepted, which would create a (horizontally dispersed) range of $x$-axis values - the SOAs - for a given fixed $y$-axis value. These SOAs could then be averaged, as was done to create the average PSS values mathematically. However, in the interests of presenting the data in their original form, the graphed data shown in the present article simply represent the response functions of participants averaged across participants by averaging the proportion of upper-tap-first responses at each SOA.

Table A1

Mean Points of Subjective Simultaneity (in Milliseconds) for Somatosensory Stimuli (Present Study) and Visual Stimuli (Shore et al. [2001] Study)

\begin{tabular}{|c|c|c|c|c|c|c|}
\hline \multirow[b]{3}{*}{ Task Type } & \multicolumn{3}{|c|}{ Somatosensory } & \multicolumn{3}{|c|}{ Visual } \\
\hline & \multicolumn{3}{|c|}{ Cue Condition } & \multicolumn{3}{|c|}{ Cue Condition } \\
\hline & Lower Tap & Neutral & Upper Tap & Vertical & Neutral & Horizontal \\
\hline \multicolumn{7}{|c|}{ Exogenous Cue } \\
\hline "Which tap first?" & 30 & 11 & -27 & 79 & -2 & -68 \\
\hline "Which tap second?" & 23 & 15 & -16 & 41 & -6 & -54 \\
\hline Average & 26 & 13 & -22 & 60 & -4 & -61 \\
\hline \multicolumn{7}{|c|}{ Endogenous Cue } \\
\hline "Which tap first?" & -34 & -48 & -55 & 32 & 4 & -28 \\
\hline "Which tap second?" & 19 & 3 & -28 & 1 & -11 & -5 \\
\hline Average & -9 & -23 & -42 & 17 & -4 & -17 \\
\hline
\end{tabular}




\section{APPENDIX (Continued)}

Using the values presented in Table A1, second-order response biases can be estimated. For somatosensory stimuli, the estimated prior entry effect calculated for the "which first" condition (half of the interval between upper- and lower-tap-cued conditions: $29 \mathrm{msec}$ ) was larger than that for the "which second" condition (20 msec) by $9 \mathrm{msec}$. The response bias (toward selecting the elevation of the attended cue) can therefore be estimated as $4.5 \mathrm{msec}$, which is half the difference between the "which first" and "which second" judgments (Shore et al., 2001, p. 205). For endogenous cuing, a reverse effect was observed: The prior entry effect for the "which second" condition ( $24 \mathrm{msec})$ was larger than that for the "which first" condition $(11 \mathrm{msec})$ by $13 \mathrm{msec}$, indicating a reverse response bias (away from selecting the elevation of the attended cue) of $6.5 \mathrm{msec}$. However, in neither the exogenous or endogenous experiment were these effects significant (as was indicated by cue condition $\times$ task type interaction). By comparison, for visual stimuli, the response bias for both exogenous and endogenous cuing was $13 \mathrm{msec}$. The PSS values derived in the study by Shore et al. are not strictly comparable because in the former study, they were derived by a method of linear interpolation, and in the latter, by a method of interpolation based on fitting cumulative normal functions to the observed data.

(Manuscript received August 14, 2008;

revision accepted for publication December 14, 2008.) 